\title{
The research and application of computer image processing
}

\author{
YunHui Qu, a \\ ${ }^{1}$ Computer Teaching and Research Section, Xi'an Medical University, Xi'an, Shaanxi Province, \\ China \\ a39358854@qq.com
}

Keywords: Computer, Image processing, Research, Application

\begin{abstract}
Research and application of image processing technology is more and more received the influence of social development, and with its own technical characteristics, in turn, affect the progress of the society as a whole technology. This paper simply summarized the recent development of digital image processing techniques and application status, enumerates the main advantages of the technology of digital image processing and the factors that restrict its development ideas at the same time the application and development of image processing technology in the future.
\end{abstract}

\section{Introduction}

Basic image processing technology can be divided into two categories: analog image processing and digital image processing. Digital image processing refers to converting image signal into digital signal and the process of using a computer for processing. Its advantage is high processing precision, processing the content is rich, complex nonlinear processing, can be flexible ability to in general as long as change the software can change the processing content [1]. Difficulties mainly on processing speed, especially for complex processing. Digital image processing techniques mainly include the following contents: geometry processing, the arithmetic processing, image enhancement, image restoration, image reconstruction, image coding, image recognition, image understanding. Digital image processing techniques involved in the development of information science, computer science, mathematics, physics and biology discipline [1], so the edge of the mathematical and related disciplines of image processing, the development of science, more and greater impact. In recent years, digital image processing technology is mature, it is widely used in space exploration, remote sensing, biomedicine, artificial intelligence and industrial testing and many other fields, and to promote these disciplines to produce a new development.

\section{Image processing technology}

The concept of image. Image contains its description information expressed by the object. We are living in an information age, the scientific research and statistics show that people get the information from the outside world about seventy percent from the visual system, which is obtained from the image, namely the photos we know, painting, animation, video, etc.

Image processing technology. Image processing technology is emphasized in the image transformation between them, the main goal is to make a various of image processing in order to improve the visual effect of image and ground for the subsequent automatic target recognition, or of image compression coding in order to reduce the space needed for image storage or the time needed for image transmission [2]. Image processing is relatively low-level operations, it is mainly on the pixel level image processing, is a large amount of data processing.

\section{Study status of computer image processing technology}

Entering the 21st century, with the rapid development of computer technology and the continuous improvement of the related theory, digital image processing technology, is accorded wide-ranging emphasis in many applications and made major pioneering achievements. Along with the computer technology and artificial intelligence, the rapid development of the thinking of scientific research, 
digital image processing to a higher and deeper development [2]. People have begun to study how to use a computer system to explain image, similar to the human visual system to understand the outside world, this is called image understanding and computer vision.

From the aspects of image transformation, the new research of wavelet transform in time domain and frequency domain has good localization characteristics, image processing, it has a broad and effective application. And image enhancement and recovery image enhancement and recovery in order to improve the quality of the image, such as removing noise, enhance image clarity, etc., mainly in the fingerprint image enhancement processing, medical imaging has a significant achievement [2]. The technology makes the spatial resolution and contrast of each image with a larger increase, and the latest medical image fusion is refers to medical image information, such as CT, MRI, SPECT, and PET images, using computer technology are integrated together, realize the information synchronization of visualization, for a variety of medical imaging play a complementary role.

Image segmentation image segmentation is one of the key technologies of digital image processing. Image segmentation is meaningful in the image are extracted from the characteristics of the part, this is the basis of the analysis of the image recognition, and understanding [3]. Although at present has developed a lot of edge detection and region segmentation method, but not a common are suitable for all kinds of effective methods for image. Therefore, the study of image segmentation are deep, is currently one of the hot topics in the study of image processing.

Image description image description is the necessary premise of image recognition and understanding. The simplest binary image can be used as its geometric characteristics describe features of the object, the general description of image methods using two-dimensional shape description, it has a boundary description and describe two kinds of methods. For the special texture image can be used $2 \mathrm{~d}$ texture feature description [3]. With the further development of the research of image processing, has begun the research described a $3 \mathrm{~d}$ object, put forward the volume method such as description, description, generalized cylinder surface description; Image classification (recognition) image classification (recognition) belongs to the category of pattern recognition, image is the main content after some pre-processing (after enhancement, recovery and compression), image segmentation and feature extraction, which sentence classification. In recent years developed a new fuzzy pattern recognition and artificial neural network pattern classification is becoming more and more attention in image recognition.

The characteristics of the image processing technology [4]. 1) Handling very informative. Digital image processing information are mostly two-dimensional information, processing very informative. As a low resolution 256 x 256 black and white image, requires about $64 \mathrm{k} / \mathrm{bit}$ amount of data; For high resolution color 512 x 512 images, insist on $768 \mathrm{k} /$ bit data volume; If you want to deal with the image sequence of 30 frames per second, per second to request $500 \mathrm{k} / \mathrm{bit} \sim 22.5 \mathrm{Mbit}$ data volume. So the computer calculation speed and high storage capacity and other requirements.

2) Takes up with wider frequency band. Digital image processing to take up the band is wide. Compared with language information, the amount of several orders of magnitude larger band. Such as the television image is about $5.6 \mathrm{MHz}$ bandwidth, and only about $4 \mathrm{KHZ}$ voice bandwidth. So in imaging, transmission, storage, processing, and display and so on each link on the implementation of technical difficulty is bigger, the cost is high, it's the band compression technology put forward higher requirements.

3) The correlation of pixels. Digital image of each pixel is not independent, its relevance. On the image, there are often many pixels with the same or close to gray. In terms of television images, two adjacent pixels in the same row or two adjacent pixels, the correlation coefficient of 0.9 above, and the correlation between two adjacent frames larger than frame correlation general said. Therefore, the potential of information compression in image processing.

4) Not all the geometry information retrieval three-dimensional scene. Because the image is a two-dimensional three-dimensional scene projection, an image itself does not have all the geometry information retrieval three-dimensional scene, it is clear that the $3 \mathrm{~d}$ scene behind some information on the $2 \mathrm{~d}$ image is reflect not to come out. Therefore, to analyze and understand the $3 \mathrm{~d}$ scene must make appropriate assumptions or additional new measurement, such as binocular image or multiple 
viewpoints. In understanding the three-dimensional scene requires knowledge guidance, this also is in the artificial intelligence is committed to solve the problem of knowledge engineering.

5) Are greatly influenced by human factors. After the digital image processing of the image is generally of observation and evaluation to the person, thus greatly influenced by human factors. Because the human visual system is very complex, environmental conditions, visual performance and impact on the person's mood hobby and the status of knowledge, as the image quality of evaluation has yet to be further in-depth study. Computer vision, on the other hand, it is an imitation of one vision, one is certainly affected the perception mechanism of the research of computer vision.

\section{Application field image technology}

Image is the main source of human and exchange information, therefore, the application of image processing areas inevitably involves all aspects of human life and work. With the expansion of the scope of human activities, the application field of image processing will be expanded.

The application of aerospace and aviation technology. Digital image processing technology applied in aerospace and aviation technology, many countries sent a lot of reconnaissance aircraft on earth area are interested in a large number of aerial photography. Analyzing from the photo processing, equipped with advanced computer image processing system is used to interpretation analysis, save manpower, and picked up speed, can also be extracted from pictures artificial can find lots of useful information [5]. Whether these images in the process of imaging, storage, transmission, or in interpretation analysis, must be a lot of digital image processing methods. Now all countries in the world in the image obtained by using Landsat resource investigation, such as forest survey, ocean survey of sediment and fisheries, water, etc.), disaster detection, such as pest detection, fire detection, environmental pollution detection, etc.), resource survey, such as oil exploration, ore output detection, large engineering exploration and analysis of geographical position, etc.), agricultural planning (e.g., soil nutrients, moisture and crop growth, yield estimation, etc.), city planning, such as geological structure, water and environmental analysis, etc.). Our country also successively carried out the above aspects of some practical applications, and have gained good effect. In meteorological forecast and other planets to space research, digital image processing technology has also played a considerable role.

Industrial and engineering applications. Image processing technology in the field of industrial and engineering has been widely used, such as automatic assembly line to detect the quality of parts, and classifying the parts, printed circuit board fault inspection, stress analysis of photo elastic mechanics, fluid mechanics, the drag and lift of the picture analysis, automatic sorting, postal mail within some poisonous and radioactive environment to identify artifacts and shape and arrangement of object state, advanced design and manufacturing technologies used in industrial vision and so on [5]. Which be worth what carry is developed with vision, hearing and touch function of intelligent robot, will bring new incentives to the industrial and agricultural production, is currently in the industrial production of spray paint, welding, assembly get effective use.

The application of image recognition technology. In coal mine production process, the use of video card to the input video signal is digitized and, through digital image feature extracting for dynamic target detection [6]. At the same time in the system the application of image processing and pattern recognition technology of image analysis, according to the result of image analysis accident alarm signal directly, or directly for fault analysis in video images. Failure occurs, the system can automatically judge malfunctioning equipment, aim the camera automatically fault equipment at the same time, displayed on the screen. This will be a high-level application of image processing technology in the future.

The application of biomedical engineering. The application of digital image processing in biomedical engineering is very extensive, and very productive. In addition to CT technique described above, and one is for the processing of medical microscopic image analysis, such as red blood cells, white blood cell classification, chromosome analysis, cancer cells recognition, etc. [6]. In addition, in the X-ray lung images norm, ultrasound image processing, e.g. analysis, stereotactic radiotherapy in a medical diagnosis of widely used image processing techniques. The example is shown in Fig.1. 


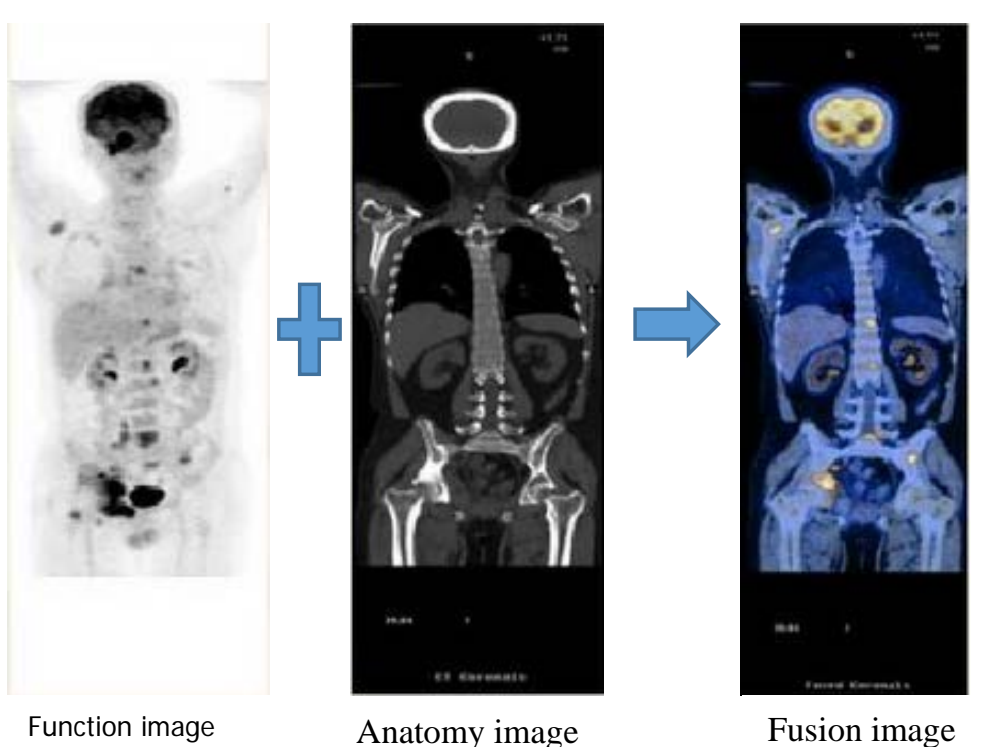

Fig.1 The application of image processing technology in medicine

The application of communication engineering. The main development direction of communication is the combinations of voice, text, images and data multimedia communication. Specifically is to telephone, TV and computer in digital communication in the form of three nets Internet transmission [7]. In image communication is the most complex and difficult, because of the amount of image data is very huge, such as transfer color TV signal at a rate of more than $100 \mathrm{mbit} / \mathrm{s}$. To such a high rate of data are transmitted in real time, coding techniques must be used to compress the information bits. In a sense, coding compression is the key to a successful these technologies. In addition to have been used more widely entropy coding, DPCM coding, transform coding, is developing the research at home and abroad new coding method, such as branch coding, adaptive network coding, wavelet transform image compression coding, etc.

Applications in military and security. Image processing and recognition in the military field is mainly used for precise missile terminal guidance, a variety of reconnaissance photo interpretation, image transmission, storage and display of military command automation system, aircraft, tanks and warship simulation training system, etc., that military technology design is shown in Fig.2 [7]. Public security business image interpretation analysis, fingerprint identification, face identification, incomplete picture of the recovery, as well as traffic monitoring, accident analysis and so on. Has been put into operation the highway without a stop automatic charging system is the vehicle and the license plate automatic recognition in the image processing technology examples of successful application.

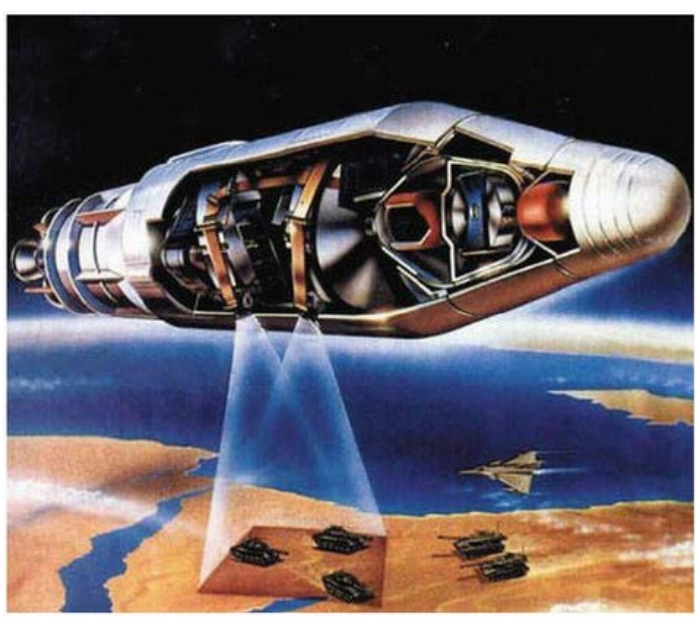

Fig.2 The application of image processing technology in military technology design

Culture and arts should be. At present this kind of application of digital television editing, animation, games, electronic image textile arts and crafts design, costume design and production, hair 
styling, data replication and repair the picture, cultural relics athletes action analysis and evaluation, etc.

\section{Summary}

Digital image processing technology in the aerospace, industrial production, medical diagnosis, resources and environment, weather and traffic monitoring, culture, education and other fields has been widely used, created huge social value; Also far cannot satisfy the needs of society, has itself in developing and perfecting, there are many new ways to explore. It will be deeper and more perfect direction first: processing algorithm better, faster processing speed, realize the graphic intelligent production, processing, recognition and understanding.

\section{References}

[1] Zh.L. Yang, Digital image technology research, People's posts and telecommunications publishing house, 2003, vol.6, pp.16-19.

[2] Y.F. Nie and L.H. Liu, The development and application of digital signal processor, Photovoltaic engineering, 2007, vol.3, pp.67 70 .

[3] Z.Z. Hou, and E.L Pao, Wavelet analysis applied research, Calculate geophysical geochemical exploration technology, 2006, vol.3, pp.6-9.

[4] D.Y. Lidao-yuan li, Chang Min, a review of the digital watermarking based on wavelet transform, Computer application and engineering, 2003, vol.6, pp.19-23.

[5] J.X. Sun, Image analysis applications, Beijing: science press, 2005, vol.3, pp.63-66.

[6] X.K. Sun and W.W. Shi, Multimedia communication technology application research, Beijing: Beijing University of posts and telecommunications publishing house, 2006, vol.4, pp.53-57.

[7] Ch.T. Liu, The application of image processing technology, Beijing: Beijing University of posts and telecommunications publishing house, 2007, vol.8, pp.47-51. 